Revista Española de

Criminológ i ca

\title{
Las Redes Bayesianas como herramienta para la evaluación del riesgo de reincidencia: Un estudio sobre agresores sexuales
}

\section{Bayesian Networks as a tool for assessing the risk of recidivism: A study on sexual offenders}

Recibido el 24 octubre 2014/Publicado el 10 diciembre 2015

\author{
Rosario Delgado ${ }^{1}$ \\ Universidad Autónoma de Barcelona
}

Xavier-Andoni Tibau

Tibau \& Ytxart Expertise

\section{RESUMEN}

La reincidencia, especialmente en delitos sexuales, constituye una problemática que afecta a los ámbitos político, jurídico-penal y social, con gran repercusión en los medios. Por este motivo es necesario desarrollar metodologías de análisis para su predicción, para mejorar la eficiencia de la gestión del riesgo asociado. Las Redes Bayesianas han demostrado ser una herramienta muy útil en la predicción de sucesos que dependen de muchas variables en un entorno de incertidumbre. Este trabajo introduce su uso como metodología novedosa en la evaluación del riesgo de reincidencia, a partir de un estudio sobre delincuentes sexuales basado en la información recogida en un informe previo. Se observa la coherencia de los resultados obtenidos con los del informe en cuanto a la influencia individual de las variables explicativas en el riesgo de reincidencia. El interés principal de esta metodología radica

\footnotetext{
${ }^{1}$ La correspondencia debe enviarse a: Rosario Delgado. Departamento de Matemáticas. Campus de la Universitat Autònoma de Barcelona (UAB). Facultad de Ciencias. Carrer del Til.lers/Avda. de l'Eix Central. 08193 Bellaterra (Cerdanyola del Vallès). Spain. delgado@mat.uab.cat
} 
en que permite explorar la influencia simultánea de diversas variables, por lo que se podrán hacer estimaciones precisas de riesgo individualizado y estudiar las interacciones entre los factores de riesgo.

Palabras Clave. Red Bayesiana, evaluación de riesgo, reincidencia, delincuencia sexual.

\begin{abstract}
Recidivism in sexual offences is a problem that affects the political, criminal justice and social context, with great media coverage. In order to improve the efficiency in managing the risk of these offences some methodologies for prediction need to be developed. Bayesian Networks have proved to be a useful tool in predicting events that depend on many variables in an uncertain environment. This paper introduces their use as a novel methodology in assessing the risk of recidivism, from a study of sex offenders based on the information in a report. Consistency of the results obtained with the report regarding the individual influence of the explanatory variables on the risk of recurrence was observed. The main interest of this methodology is to explore the simultaneous influence of several variables, so it may make accurate estimates of individual risk and study the interactions between the risk factors.
\end{abstract}

Key Words. Bayesian Network, risk assessment, recidivism, sexual offences.

\title{
1. Introducción
}

Los casos de agresiones, especialmente de agresiones sexuales, sobre todo si se trata de un agresor reincidente, son vistos por las instituciones y la sociedad en general como una amenaza cada vez más mayor (La Fond, 2005). En la actualidad predominan las teorías mixtas del Derecho Penal, que integran tanto las teorías retributivas o absolutas como las relativas, y según las cuales las condenas impuestas a los delincuentes en general (y a los delincuentes sexuales en particular) deben servir para su reinserción y no únicamente para sancionarlos y apartarlos temporalmente de la sociedad (teorías retributivas). Por ello, la investigación criminológica se ha ocupado en los últimos años del diseño de herramientas tales como protocolos de evaluación y escalas que permiten valorar el riesgo de cometer delitos sexuales (Andrés-Pueyo \& Echeburúa, 2010), con el objetivo de proporcionar información valiosa para tratar de responder a las preguntas (Hanson, 1998): ¿cuál es la probabilidad de que un agresor sexual cometa un nuevo 
delito? ¿qué se puede hacer para reducir esta probabilidad? ¿puede reducirse mediante un tratamiento adecuado? ¿qué características debe tener para que el tratamiento sea efectivo?

Estas son las cuestiones clave de la investigación criminológica en este contexto, a las que algunos estudios intentan dar respuesta (Gendreau, Little \& Goggin, 1996; Redondo, 2006; Redondo \& Garrido, 2008). Aunque es imposible eliminar completamente el riesgo de reincidencia, hay que fomentar aquellas actuaciones e intervenciones encaminadas a su reducción, y para ello es necesario mejorar las metodologías existentes para la evaluación del riesgo de reincidencia, así como desarrollar nuevos procedimientos que las complementen. A pesar de la alarma social que generan, que se explica por la naturaleza del delito, resulta que los delincuentes sexuales reinciden menos que los no sexuales (Grubin \& Wingate, 1996). La literatura coincide en cifrar la reincidencia de los delincuentes sexuales, cuando no se especifica el tipo de delito, en torno al 35\%. Si tenemos en cuenta el tipo de reincidencia, la tasa se sitúa entre el $14 \%$ y el $20 \%$ pasados los 5 años (Hanson \& Morton-Bourgon, 2004; Harris \& Hanson, 2004; Lösel, 2002; Quinsey, Lalumière, Rice \& Harris, 1995).

Profundizando más vemos que la reincidencia no es homogénea sino que depende tanto de la tipología de delito como del perfil del delincuente (Hanson \& Bussière, 1998; Hanson \& Morton-Bourgon, 2004). El modelo del Triple Riesgo Delictivo (TRD) define factor de riesgo como cualquier elemento personal, familiar o social que, de acuerdo con la investigación, se asocia a un mayor riesgo delictivo, y como factor de protección aquél que reduce el riesgo (Redondo, 2008). Los factores de riesgo pueden ser a su vez dinámicos o estáticos (Andrews \& Bonta, 2010). Los estáticos son aquéllos invariables, como por ejemplo la edad del primer ingreso en prisión, mientras que los dinámicos se pueden modificar mediante intervención, como estar o no incluido en un programa de control de agresiones sexuales. Esto evidencia la necesidad de desarrollar herramientas que permitan ahondar en la comprensión de las variables explicativas de la reincidencia, para ver cuáles son factores de riesgo que incrementan la tasa de reincidencia, y cuáles no, y así poder prevenirla incidiendo sobre los factores de riesgo dinámicos (Lalumière \& Quinsey, 1996; Quinsey, Rice \& Harris, 1995). 
En este artículo presentamos una metodología ampliamente conocida y utilizada en muy diversos ámbitos para la evaluación de riesgos en general pero novedosa, hasta donde sabemos, en la evaluación del riesgo de reincidencia de delincuencia en España: las Redes Bayesianas. Aunque la opinión de los expertos es, y seguirá siendo, fundamental en la valoración de la importancia de los diferentes factores de riesgo en la reincidencia delictiva, es preciso introducir metodologías basadas en herramientas cuantitativas que ayuden a los expertos en la evaluación de riesgos. Esta evaluación es de vital importancia ya que, según el principio fundamental de riesgo establecido por Andrews \& Bonta (1998), el tratamiento de los delincuentes debe modularse de acuerdo con el nivel de riesgo de cada individuo, de manera que aquéllos que presentan un riesgo más alto reciban una intervención más intensiva.

Las Redes Bayesianas fueron introducidas en la década de 1920 como herramienta gráfica que describe el conocimiento probabilístico de las relaciones entre variables que afectan a un determinado fenómeno no determinista. Desde entonces han demostrado su enorme utilidad en los procedimientos de toma de decisión en un gran número de campos, y su uso para la evaluación de riesgos está ganando popularidad día a día e incluye aplicaciones en áreas tan diversas como la economía (Adusei-Poku, 2005), la medicina (Cruz-Ramírez, Acosta-Mesa, Carrillo-Calvet, Nava-Fernández \& Barrientos-Martínez, 2007), el riesgo medioambiental (Pollino, Woodberry, Nicholson, Korb \& Hart, 2007), los desastres ecológicos (Ticehurst, Newham, Rissik, Letcher \& Jakeman, 2007) o los accidentes con residuos nucleares (Lee \& Lee, 2006). En el ámbito forense, las Redes Bayesianas han alcanzado cierta notoriedad en relación con algunos casos famosos de la justicia norteamericana, como el de People vs. Collins o el de los anarquistas Sacco y Vanzetti, ejecutados in 1927 (Aitken \& Taroni, 2004), o más recientemente, el caso de O.J. Simpson (Olumide, 2010), e incluso se han utilizado para presentar evidencias legales ante un tribunal de justicia (Fenton \& Neil, 2010).

Basándonos en estos antecedentes, pensamos que las Redes Bayesianas pueden aportar un enfoque novedoso desde una óptica tanto práctica como teórica. Su facultad para mostrar cómo interactúan las variables entre ellas, cómo se potencian o se atenúan unas a otras, debería permitir en futuras investigaciones analizar el porqué de estas interacciones y así esclarecer la naturaleza de las mismas. Paralelamente, su capacidad para estudiar el efecto de los diversos factores de riesgo permite establecer perfiles de 
riesgo de reincidencia más realistas, adaptados a cada sujeto, que contemplen la interacción entre los factores.

En este trabajo presentamos las Redes Bayesianas como una herramienta para la identificación de factores de riesgo de reincidencia y su evaluación, mediante un estudio que utiliza la información contenida en un informe previo ${ }^{2}$ en el que se analizaron los datos de reincidencia de delincuentes sexuales de cárceles catalanas. A partir de esta información construimos una Red Bayesiana ad hoc y analizamos los resultados estadísticos sobre reincidencia que nos proporciona, comparándolos con las conclusiones del informe previo y de otros trabajos publicados relacionados.

\section{Método}

Nuestro trabajo utiliza la información que aporta un informe previo referido a internos penitenciarios de cárceles catalanas que habían sido condenados por delitos relacionados con la agresión sexual. El informe se basa en un estudio en el que a lo largo de tres años, se tuvieron en cuenta algunas variables relacionadas con la reincidencia con el objetivo de ayudar en la evaluación de su riesgo y, especialmente, determinar cuáles son los factores significativos para la reincidencia de los delincuentes sexuales.

Los individuos del estudio correspondían a una muestra seleccionada de la base de datos de los centros penitenciarios catalanes SIPC (Sistema Informàtic Penitenciari Català), formada por internos condenados por un delito de tipo sexual que salieron de prisiones catalanas entre el 1 de Enero de 1998 y el 31 de Diciembre de 2003, tanto en libertad definitiva por haber cumplido la totalidad de la condena, como en cualquiera de las modalidades de la libertad condicional: un total de 315 individuos a los que se les hizo un seguimiento hasta el 1 de Noviembre de 2007.

En el presente trabajo, como en el informe previo, consideramos que delitos sexuales son aquéllos comprendidos en el título VIII del vigente Código Penal y que son clasificados como "delitos contra la libertad y la indemnidad sexual", exceptuando

\footnotetext{
${ }^{2}$ Documents de Treball, Investigació: "Delictes sexuals i reincidència. Un estudi a les presons de Catalunya. Avaluació i predicció del risc de reincidència en agressors sexuals. Recomanacions de la comissió per a l'estudi de les mesures de prevenció en la reincidència en delictes greus" (2009). Àmbit social i criminològic. Centre d'estudis jurídics i formació especialitzada. Departament de Justícia. Generalitat de Catalunya.
} 
los delitos de corrupción de menores. Teniendo en cuenta que en estas investigaciones están incluidos sujetos condenados en aplicación del anterior Código Penal, se incluyen también los delitos tipificados según esta ley que cumplen las mismas características que los anteriores.

\subsection{Las variables del estudio}

Las variables que consideramos en el estudio son las seleccionadas en el informe: una variable explicada llamada Reincidencia y once variables explicativas escogidas en función de su potencial efecto sobre el riesgo de reincidencia de los delincuentes sexuales. Según su tipología, estas variables explicativas se pueden clasificar en:

(i) Relativas a la carrera delictiva: Antecedentes, Versatilidad, Tipo de víctima, Número de delitos sexuales.

(ii) Relativas al fracaso de las medidas de supervisión en la comunidad: Incumplimiento de la supervisión.

(iii) Relativas a la conflictividad durante el cumplimiento de la condena: Faltas, Régimen cerrado.

(iv) Relativas a la participación en el programa de Control de la agresión sexual SAC: Seguimiento del Programa, Valoración del seguimiento.

(v) Relativas a la condena: Régimen de vida, Tipo de libertad.

Cualquier ingreso en prisión en el intervalo de tiempo comprendido desde la salida en libertad de la condena base del preso hasta el momento en que finaliza la recogida de datos de la investigación (1 de Noviembre de 2007) se considera reincidencia. Los posibles valores de la variable explicada, según si se produce reincidencia o no, y de qué tipo, son: sexual, violenta (no sexual), general (ni violenta, ni sexual), sin reincidencia.

En este artículo presentamos una versión simplificada del estudio que hemos realizado, en la que de las variables explicativas, sólo vamos a considerar dos, con el fin de ejemplificar el uso de las Redes Bayesianas en la evaluación del riesgo de reincidencia. Ambas variables son relativas a la carrera delictiva y son consideradas factores de especial riesgo, tanto el Tipo de víctima (Hanson \& Bussière, 1998) como la 
Versatilidad delictiva (Hanson \& Morton-Bourgon, 2005). Sus posibles valores son: Versatilidad: Sí (existen penas antes o durante la condena base por cualquier tipo de delito no sexual) o No. Tipo de víctima: mujeres, intrafamiliar, chicos extrafamiliar ( $<18$ años) o chicas extrafamiliar ( $<18$ años).

\subsection{Las Redes Bayesianas}

Las Redes Bayesianas son un modelo matemático gráfico útil en contextos no deterministas en los que intervienen diversas variables, adecuado para representar las relaciones entre ellas. En nuestro caso, las variables que intervienen son la variable explicada Reincidencia y las once variables explicativas comentadas en la Sección 2.1, aunque en este artículo presentamos un modelo simplificado con sólo dos de ellas a modo de ejemplo: Versatilidad y Tipo de víctima.

En las Redes Bayesianas las variables se representan mediante nodos en un grafo, y las relaciones entre ellas (no necesariamente del tipo causa-efecto) se representan mediante flechas.

Para evaluar el riesgo de reincidencia de los delincuentes sexuales construiremos una Red Bayesiana ad hoc que para cada delincuente sexual, en función de las variables explicativas, nos permita obtener su probabilidad (tasa) de reincidencia, tanto sexual como violenta (no sexual) o general (ni violenta ni sexual). El cálculo de esta probabilidad se puede llevar a cabo bajo ciertas suposiciones de independencia y se basa en el uso del conocido Teorema de Bayes (Bayes, 1763). Cuando la red consta de diversos nodos, la complicación de los cálculos hace necesario llevarlos a cabo con la ayuda de algún programa (en este artículo hemos usado Hugin Lite ${ }^{3}$ ).

A partir de la información contenida en el informe previo obtenemos las probabilidades a priori de la variable Reincidencia (R), que suman 1 (en porcentaje, 100\%), y podemos ver en la Tabla 1.

\footnotetext{
${ }^{3}$ Hugin Lite 7.8 es una versión demo gratuita del programa Hugin Developer / Hugin Researcher. Puede bajarse de: http://www.hugin.com/productsservices/demo/hugin-lite.
} 
Tabla 1. Probabilidades a priori del nodo Reincidencia

\begin{tabular}{|l|r|}
\hline \multicolumn{1}{|c|}{ Reincidencia } & \multicolumn{2}{|c|}{ Porcentaje } \\
\hline Sexual & $5.80 \%$ \\
\hline Violenta & $6.50 \%$ \\
\hline General & $6.20 \%$ \\
\hline Sin reincidencia & $81.50 \%$ \\
\hline
\end{tabular}

Observemos que el riesgo de reincidencia sexual de los delincuentes sexuales no es especialmente alto comparado con los otros tipos de reincidencia (violenta y general); de hecho, es ligeramente menor. Veremos en el apartado Discusión que esta afirmación se puede matizar gracias a la Red Bayesiana.

El Anexo 1 ilustra el uso del Teorema de Bayes a partir del caso más simple posible de Red Bayesiana.

La red Bayesiana que presentamos en este artículo consta de 3 nodos, el de la variable explicada Reincidencia, y los correspondientes a las variables explicativas, a los que les llega una flecha del nodo Reincidencia, como muestra el grafo de la Figura 1, así como de las probabilidades de las tablas 1 y 2 y la Tabla 6 del Anexo 1.

Tabla 2. Probabilidades condicionadas del nodo Tipo de víctima

\begin{tabular}{|l|c|c|c|c|}
\hline \multirow{2}{*}{ Tipo de víctima } & \multicolumn{4}{|c|}{ Reincidencia } \\
\cline { 2 - 5 } & Sexual & Violenta & General & Sin reincidencia \\
\hline Mujeres & $53.80 \%$ & $83.38 \%$ & $60.00 \%$ & $49.40 \%$ \\
\hline Intrafamiliar & $0.00 \%$ & $8.31 \%$ & $20.00 \%$ & $24.70 \%$ \\
\hline Chicos extrafamiliar & $30.80 \%$ & $0.00 \%$ & $20.00 \%$ & $10.10 \%$ \\
\hline Chicas extrafamiliar & $15.40 \%$ & $8.31 \%$ & $0.00 \%$ & $15.80 \%$ \\
\hline
\end{tabular}


Análogamente a como se hace para un Red Bayesiana con dos nodos (véase la Figura 6 de Anexo 1), obtenemos las probabilidades que muestra la Figura 1.

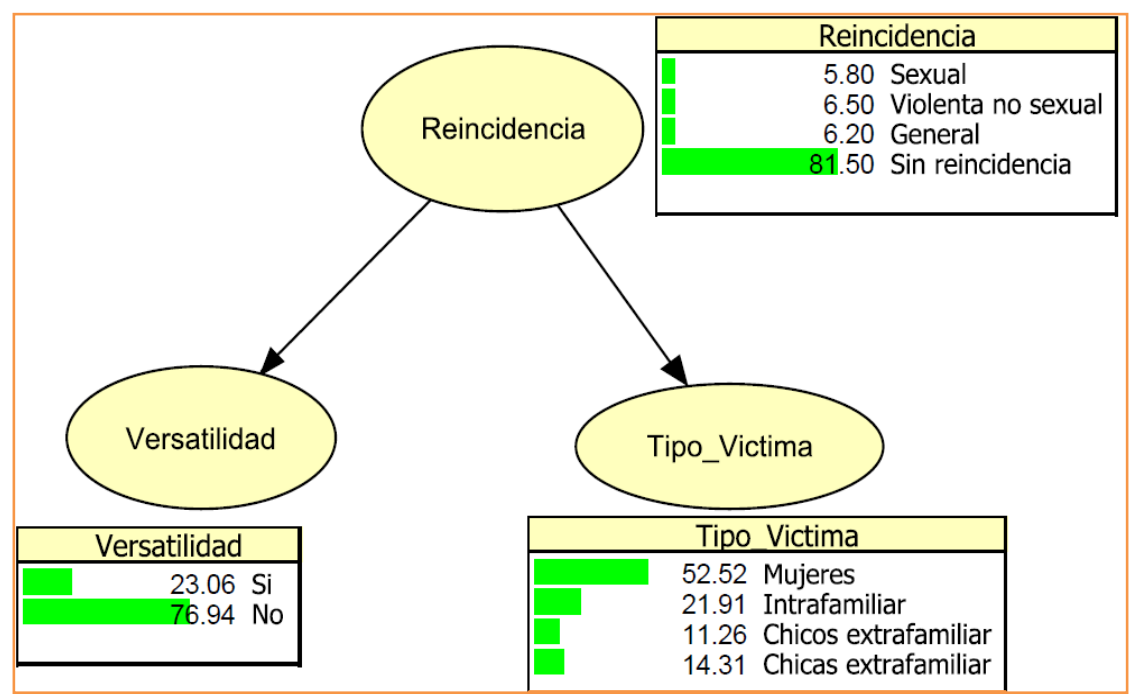

Figura 1. Grafo y probabilidades de la Red Bayesiana simplificada con tres nodos.

\section{Resultados}

La Red Bayesiana construida (Figura 1 más tablas 1 y 2 y Tabla 6 del Anexo 1) nos permite introducir evidencias para actualizar los porcentajes de la Tabla 1 mediante el proceso de Propagación Bayesiana, como vimos en Anexo 1 (Figura 7). Es decir, nos permite evaluar el riesgo de reincidencia a posteriori a partir de las evidencias expresadas en términos de las variables explicativas, obteniendo conclusiones sobre los perfiles de los delincuentes reincidentes. La situación más sencilla, considerada en el Anexo 1, corresponde a evaluar el riesgo de reincidencia a posteriori a partir de una evidencia expresada en términos de una sola de las variables explicativas, aunque análogamente se puede trabajar con evidencias en función de dos o más variables explicativas, lo que permite estudiar su influencia conjunta en el riesgo de reincidencia, valorando su posible interacción. 


\subsection{Riesgo de Reincidencia a posteriori a partir de una evidencia en función de la variable explicativa "Versatilidad"}

La Propagación Bayesiana nos permite calcular los riesgos de reincidencia a posteriori a partir de los riesgos a priori si tenemos la evidencia de que el delincuente sexual es versátil. Análogamente obtendríamos los riesgos de reincidencia a posteriori si tuviésemos la evidencia de que el preso no lo es. La Tabla 3 nos resume los resultados obtenidos en ambos casos.

Tabla 3. Comparativa de riesgos de reincidencia a posteriori según Versatilidad

\begin{tabular}{|l|c|c|c|c|}
\hline \multirow{2}{*}{ Evidencia } & \multicolumn{4}{|c|}{ Reincidencia } \\
\cline { 2 - 5 } & Sexual & Violenta & General & Sin reincidencia \\
\hline $\begin{array}{l}\text { Sin evidencia (riesgo a } \\
\text { priori) }\end{array}$ & $5.80 \%$ & $6.50 \%$ & $6.20 \%$ & $81.50 \%$ \\
\hline Versatilidad=Sí & $16.17 \%$ & $14.91 \%$ & $15.56 \%$ & $53.36 \%$ \\
\hline Versatilidad=No & $2.69 \%$ & $3.98 \%$ & $3.39 \%$ & $89.94 \%$ \\
\hline
\end{tabular}

Y la Figura 2 nos permite interpretarlos gráficamente: la versatilidad se presenta como una dimensión del riesgo, actuando como factor de protección el hecho de no ser un delincuente versátil, mientras que es un factor de riesgo el serlo, para los tres tipos de reincidencia (sexual, violenta y general). Se observa que ser versátil provoca un incremento en el riesgo mayor que el descenso producido por la evidencia de no serlo. 


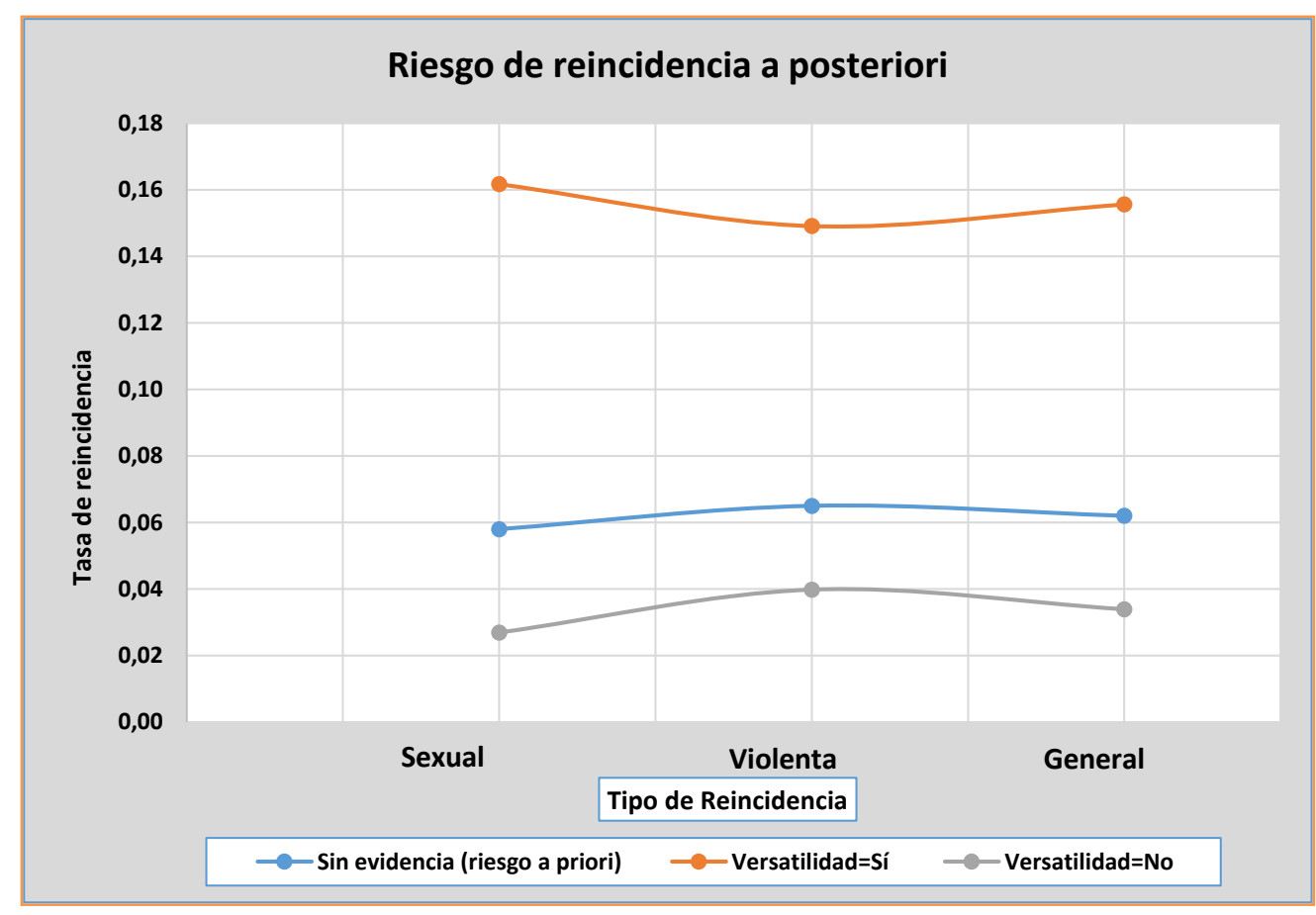

Figura 2. Comparativa de riesgos a posteriori según Versatilidad.

\subsection{Riesgo de Reincidencia a posteriori a partir de una evidencia en función de la variable explicativa "Tipo de víctima"}

Si usamos la propagación bayesiana fijando como evidencia que el Tipo de víctima es un chico ajeno al entorno familiar, observamos que el riesgo de reincidencia sexual pasa del valor a priori 5.80\% (Tabla 1), a un valor a posteriori de casi el triple, 15.87\%, como muestra la Figura 3. En cambio, el riesgo de reincidencia general pasa de 6.20\% (Tabla 1) a casi el doble, $11.01 \%$, y el riesgo de reincidencia violenta (no sexual) disminuye de $6.50 \%$ (Tabla 1) a $0.00 \%$. Esto último probablemente se deba a que exista cierta tendencia en los delincuentes sexuales cuyas víctimas son chicos ajenos al entorno familiar, a reincidir con el mismo tipo de delito en vez de hacerlo con delitos de otra índole (Langevin et al., 2006). 


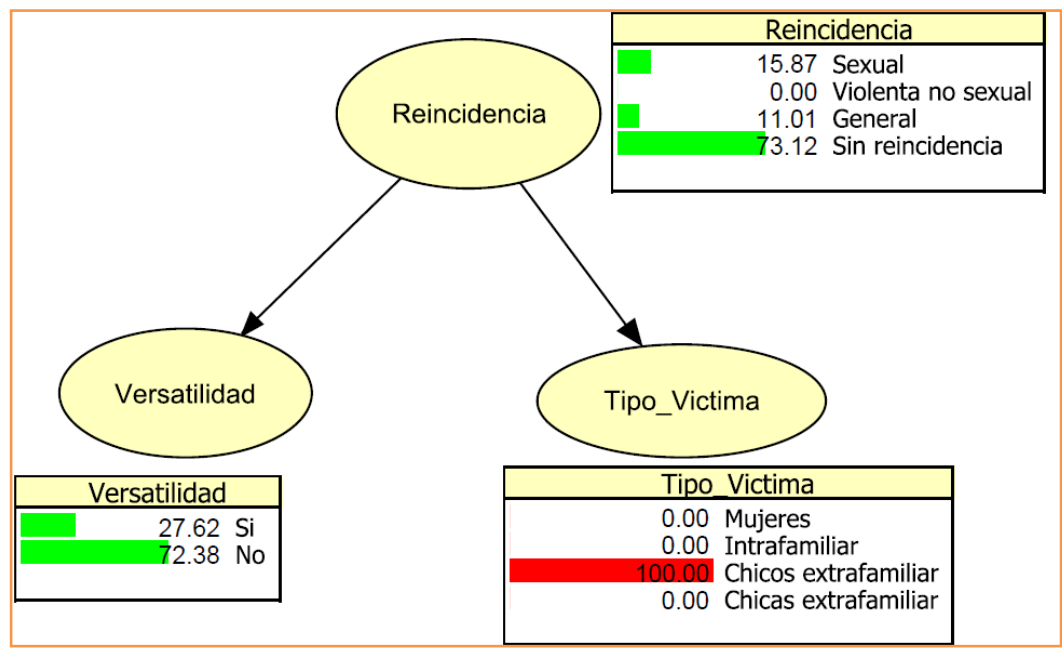

Figura 3. Riesgo de reincidencia a posteriori con Evidencia: Tipo de víctima = chico extrafamiliar.

$\mathrm{Si}$ vamos introduciendo como evidencia los diferentes tipos de víctima, obtenemos los riesgos a posteriori que la Tabla 4 recoge, ordenándolos de mayor a menor (los que no aparecen son cero).

Tabla 4. Comparativa de riesgos de reincidencia a posteriori según el Tipo de víctima

\begin{tabular}{|l|l|l|}
\hline \multicolumn{2}{|c|}{ Sexual } & \multicolumn{1}{c|}{ Veincidencia } \\
\hline $\begin{array}{l}\text { Chicos extrafamiliar } \\
\mathbf{1 5 . 8 7 \%}\end{array}$ & Mujeres 10.32\% & $\begin{array}{l}\text { General } \\
\text { Chicos extrafamiliar } \\
\text { Chicas extrafamiliar }\end{array}$ \\
6.24\% & $\begin{array}{l}\text { Chicas extrafamiliar } \\
\text { Mujeres 5.94\% }\end{array}$ & Mujeres $7.08 \%$ \\
\hline
\end{tabular}

A partir de la Tabla 4 podemos ver gráficamente la comparativa de riesgos a posteriori, según el tipo de víctima, en la gráfica central de la Figura 5. En efecto, observamos que el tipo de delincuente sexual que más reincide en delitos sexuales es el que ha abusado previamente de chicos ajenos al entorno familiar, seguido por el grupo 
de agresores de chicas fuera del entorno familiar y el de mujeres, con prácticamente la misma tasa de reincidencia. Por tanto, que la víctima sea un chico ajeno al entorno familiar es un factor de riesgo para la reincidencia sexual, mientras que el hecho de que la víctima sea del entorno familiar reduce el riesgo de reincidencia sexual.

Por otra parte, los agresores sexuales de mujeres son los que más riesgo tienen de reincidir cometiendo delitos violentos: que la víctima del delito sexual de la condena base sea una mujer es una característica que incrementa el riesgo de reincidencia violenta, mientras que si es un chico ajeno al entorno familiar lo minimiza.

Finalmente, el riesgo que tienen los agresores sexuales de chicos ajenos al entorno familiar de reincidir con un delito general (ni sexual ni violento) es el más alto, siendo este tipo de víctima una característica que incrementaría el riesgo de reincidencia de tipo general, mientras que si la víctima es una chica ajena al entorno familiar, el riesgo de reincidencia general es mínimo.

\subsection{Riesgo de Reincidencia a posteriori a partir de una evidencia en función de las dos variables explicativas "Tipo de víctima" y "Versatilidad"}

Como hemos comentado, podemos incorporar evidencias basadas en dos (o más) variables explicativas a la red, y observar cómo afectan simultáneamente a las probabilidades a posteriori. Por ejemplo, Versatilidad=Sí y Tipo de víctima=chico extrafamiliar. 


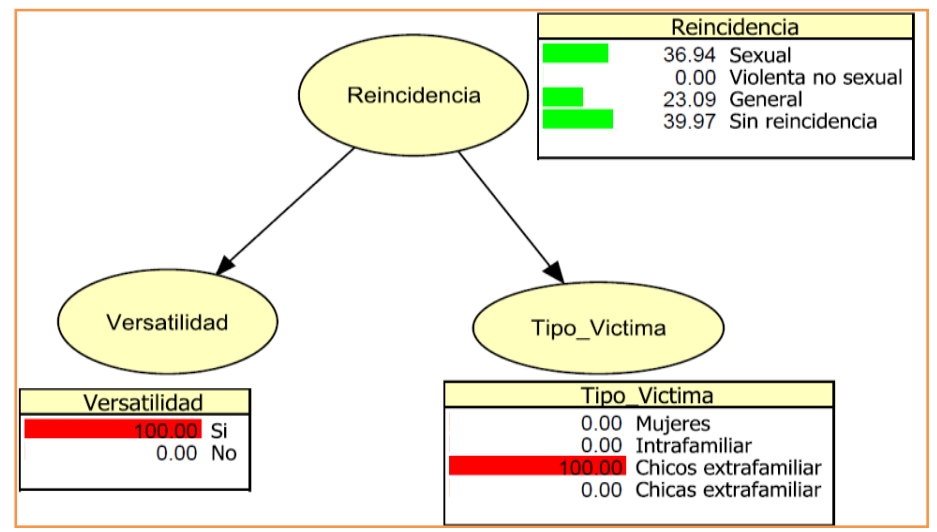

Figura 4. Riesgo de reincidencia a posteriori con Evidencia: Versatilidad=Sí y Tipo de víctima $=$ chico extrafamiliar

En la Figura 4 vemos que los riesgos de reincidencia a posteriori a partir de esta evidencia son $36.94 \%$ (sexual), $0.00 \%$ (violenta) y $23.09 \%$ (general). En la Tabla 5 hemos resumido los riesgos de reincidencia a posteriori a partir de todas las posibles evidencias relativas a las variables Versatilidad y Tipo de víctima.

Tabla 5. Comparativa de riesgos de reincidencia a posteriori según Tipo de víctima y Versatilidad

\begin{tabular}{|l|l|c|c|c|}
\hline \multirow{2}{*}{ Versatilidad } & \multirow{2}{*}{ Tipo de Víctima } & \multicolumn{3}{|c|}{ Reincidencia } \\
\cline { 3 - 5 } & & Sexual & Violenta & General \\
\hline \multirow{3}{*}{ Sí } & Mujer & $15.31 \%$ & $\mathbf{2 1 . 8 8 \%}$ & $16.43 \%$ \\
\cline { 2 - 5 } & Intrafamiliar & $\mathbf{0 . 0 0 \%}$ & $7.07 \%$ & $17.76 \%$ \\
\cline { 2 - 5 } & Chico extrafamiliar & $\mathbf{3 6 . 9 4 \%}$ & $\mathbf{0 . 0 0 \%}$ & $\mathbf{2 3 . 0 9 \%}$ \\
\cline { 2 - 5 } & Chica extrafamiliar & $20.48 \%$ & $10.19 \%$ & $\mathbf{0 . 0 0 \%}$ \\
\hline \multirow{3}{*}{ No } & Mujer & $2.83 \%$ & $\mathbf{6 . 4 8 \%}$ & $3.97 \%$ \\
\cline { 2 - 5 } & Intrafamiliar & $\mathbf{0 . 0 0 \%}$ & $1.42 \%$ & $2.92 \%$ \\
\cline { 2 - 5 } & Chico extrafamiliar & $\mathbf{7 . 8 3 \%}$ & $\mathbf{0 . 0 0 \%}$ & $\mathbf{6 . 4 1 \%}$ \\
\cline { 2 - 5 } & Chica extrafamiliar & $2.77 \%$ & $2.21 \%$ & $\mathbf{0 . 0 0 \%}$ \\
\hline
\end{tabular}

La interacción entre las dos variables queda ahora de manifiesto. En efecto, en la Tabla 5 vemos que los incrementos del riesgo de reincidencia observados en la Tabla 4 , 
en realidad sólo se producen en el caso de que el delincuente sexual sea versátil. Si nos centramos en los delincuentes que tienen como tipo de victima chicos extrafamiliares, observamos que el riesgo de reincidencia sexual cuando el delincuente es versátil casi quintuplica el mismo cuando no lo es. En cuanto a la reincidencia general, en este caso casi se cuadriplica el riesgo de reincidencia cuando el preso es versátil, mientras que el riesgo de reincidencia violenta es nulo tanto si el preso es versátil como si no. En cambio, los tipos de víctima para los que el riesgo de reincidencia era nulo (no aparecían en la Tabla 4), se mantienen independientemente de la versatilidad del delincuente. Por otro lado, según vimos en la Tabla 3, la versatilidad es un factor de riesgo para todos los tipos de reincidencia, y la no versatilidad un factor de protección; a partir de la Tabla 5 vemos que, efectivamente, esto se mantiene independientemente del tipo de víctima (exceptuando el caso de un tipo de víctima específico para cada tipo de reincidencia, el que proporciona un riesgo a posteriori nulo, tanto si el delincuente es versátil como si no).

Estos riesgos de reincidencia a posteriori han sido representados en la Figura 5. 


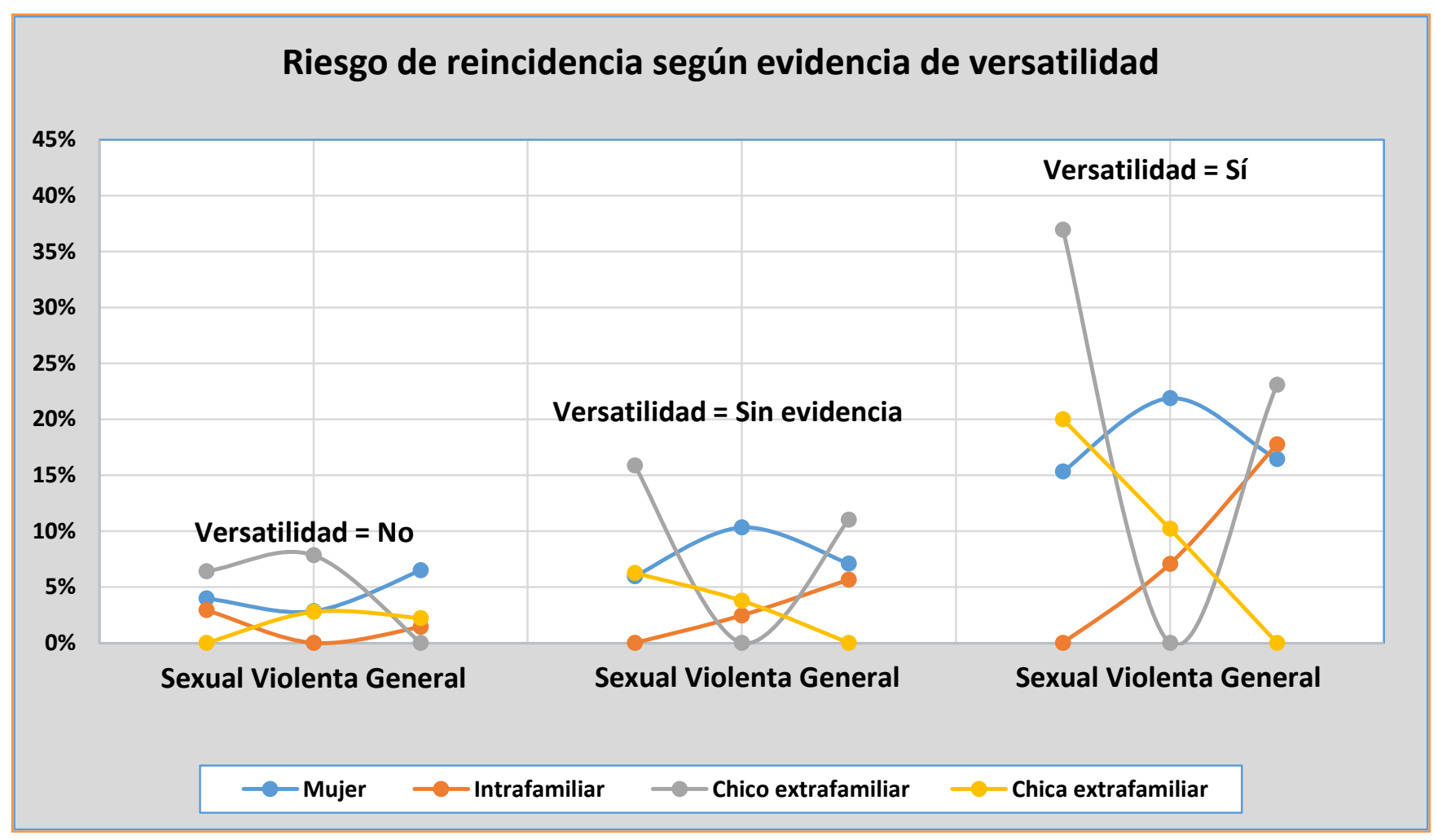

Figura 6. Comparativa de riesgos a posteriori según Tipo de víctima, con la evidencia Versatilidad=No, sin evidencia, o con la evidencia Versatilidad=Sí.

La Figura 5 evidencia gráficamente lo que la Red Bayesiana nos ha permitido constatar: el gráfico central corresponde a aquella situación en la que no tenemos evidencia sobre la versatilidad, mientras que si sabemos que no hay versatilidad (gráfico de la izquierda) vemos cómo esto afecta al riesgo de reincidir según el tipo de víctima y de reincidencia, actuando como factor de protección, aunque no en todos los casos. Sin embargo, si tenemos la evidencia de que sí hay versatilidad (gráfico de la derecha), vemos que ésta actúa generalmente como factor de riesgo, muy especialmente para el caso en que la víctima sea un chico ajeno al entorno familiar y el tipo de reincidencia sea sexual.

Observamos, en efecto, que el tipo de víctima que más incrementa el riesgo de algún tipo de reincidencia es el chico ajeno al entorno familiar, tratándose en concreto de la reincidencia de tipo sexual, resultado que concuerda con trabajos que constatan que las desviaciones sexuales como la pedofilia incrementan el riesgo de reincidencia (Hanson \& Bussière, 1998; Quinsey, Lalumière et al., 1995; Roberts, Doren \& Thornton, 2006). 


\section{Discusión}

En este artículo presentamos las Redes Bayesianas como metodología cuantitativa novedosa para el análisis y la evaluación del riesgo de reincidencia delictiva en general, a partir de un estudio sobre delincuentes sexuales. Una Red Bayesiana es un modelo probabilístico gráfico que representa las relaciones existentes entre las variables que afectan a cierto fenómeno no determinista. En nuestro caso, el fenómeno es la reincidencia de los delincuentes sexuales, y las variables consideradas en el modelo son una variable explicada, que es la Reincidencia, y las variables explicativas relativas al expediente penitenciario del delincuente, de las que consideramos sólo dos en la versión simplificada del estudio que presentamos en el artículo. A partir de la información que nos proporciona un informe previo relativo a delincuentes sexuales que han cumplido condena en cárceles catalanas, obtenemos el riesgo (probabilidad) de reincidencia a priori, tanto sexual como violenta o general, y las probabilidades condicionadas de las variables explicativas a la variable explicada. A partir de estas probabilidades construimos una Red Bayesiana ad hoc que nos permite estimar el riesgo de reincidencia a posteriori cuando disponemos de alguna evidencia sobre el delincuente sexual, expresada en términos de las variables explicativas.

Esta Red Bayesiana, aunque es preliminar y debería someterse a futuras revisiones y mejoras, permite hacer una valoración de las variables explicativas en tanto que factores de riesgo para la reincidencia delictiva de los delincuentes sexuales, ya sea de forma individual o conjuntamente (en parejas o en grupos de más de dos variables). En este sentido, los resultados que se obtienen pueden ayudar a establecer perfiles de delincuentes sexuales reincidentes con necesidades específicas de intervención, al tiempo que permiten analizar cómo estas variables interaccionan. Por tanto, la Red Bayesiana es un modelo de evaluación del riesgo de reincidencia en el que las diversas variables explicativas interaccionan entre sí y, a su vez, con la variable Reincidencia, a diferencia de aquellos modelos que no recogen la interacción y en los que los factores con puntuaciones opuestas se pueden contrarrestar.

Las conclusiones del artículo son consistentes con el informe previo y otras investigaciones consultadas, cuyos resultados complementan y matizan. Por ejemplo, 
aunque el riesgo de reincidencia a priori de los delincuentes sexuales es prácticamente igual para los tres tipos de reincidencia (sexual, violenta o general), como muestra la Tabla 1, la Figura 3 nos muestra claramente que si sus víctimas son chicos ajenos al entorno familiar, los delincuentes sexuales tienen mayor riesgo de reincidencia precisamente en delitos sexuales, lo que corresponde a un perfil de delincuente sexual muy concreto: un pederasta que actúa sobre chicos ajenos a su entorno familiar (Murray, 2000). También vemos que cuando la víctima pertenece al seno familiar, el riesgo de reincidencia sexual es nulo, lo que podría explicarse porque en este caso el delincuente sexual pierde la oportunidad de reincidir al salir de prisión.

En el informe previo también se comentaba que cuando se trata de reincidencia en delitos no sexuales, son los agresores de mujeres adultas los que tienen mayor tasa de reincidencia. Según vemos en la Figura 5, esto se cumple para el caso de la reincidencia en delitos violentos, pero no así para la reincidencia en delitos de tipo general (ni sexual ni violento), en cuyo caso el mayor riesgo de reincidencia lo tienen de nuevo los agresores de chicos ajenos al entorno familiar.

Según Soler y García (2007), la versatilidad delictiva es uno de los factores de riesgo más importante, junto con la edad cuando se produce el primer ingreso en prisión. Al analizar conjuntamente dos variables hemos observado que la versatilidad delictiva es, efectivamente un factor de riesgo importante, aunque no afecta por igual a todos los delincuentes ni tipos de víctima dado que, por ejemplo, incrementa más el riesgo de reincidencia cuando la víctima es un chico ajeno al entorno familiar, si se trata de reincidencia de tipo sexual o general, resultado que sería novedoso (Figura 5).

Algunos autores (Hanson \& Bussière, 1998; Hanson \& Morton-Bourgon, 2004) analizan la relación entre numerosos factores de relevancia criminológica y la reincidencia delictiva, llegando a la conclusión de que la reincidencia sexual y la general no tienen la misma configuración de factores de riesgo, siendo que en el caso de la reincidencia sexual, los marcadores de riesgo de primer orden son los que tienen relación con la carrera delictiva, especialmente la orientación sexual desviada hacia los menores (mayoritariamente de sexo masculino) ajenos al entorno familiar, lo que concuerda con los resultados que hemos obtenido. En general, los resultados obtenidos usando la Red Bayesiana que hemos construido nos han permitido llegar a conclusiones coherentes con la bibliografía existente, y al mismo tiempo, matizar y concretar más 
cómo las diversas variables explicativas consideradas afectan al riesgo de reincidencia, tanto por separado como conjuntamente, teniendo en cuenta sus posibles interacciones.

Esta herramienta, que es un indicador dinámico del riesgo de reincidencia delictiva construido a partir de la información de que hemos dispuesto sobre la población reclusa de delincuentes sexuales en Cataluña, permite evaluar de manera individualizada el riesgo de reincidencia así como analizar qué variables explicativas actúan como factores de riesgo en cada caso y cómo interaccionan. De esta manera, se podría disponer de una información muy valiosa para planificar actuaciones encaminadas a la reducción de la reincidencia y al desistimiento.

\section{Agradecimientos}

Los autores deseamos agradecer al editor y a los evaluadores anónimos su concienzuda lectura de una versión previa del artículo y sus pertinentes observaciones y sugerencias, que nos han permitido redactar esta versión del artículo mejorada en cuanto a su calidad comunicativa.

\section{Financiación}

Rosario Delgado recibe apoyo del proyecto MEC ref. MTM2012-33937 y FEDER "Una manera de hacer Europa". Los autores declaran que no existe conflicto de intereses o ético relacionado con el desarrollo de este trabajo. 


\section{Referencias}

Adusei-Poku, K. (2005). Operational Risk management -Implementing a Bayesian Network for Foreign Exchange and Money Market Settlement. Tesis doctoral sin publicar. Göttingen University. Disponible en :

http://www.statistics.unigoettingen.de/fileadmin/cfs/Dokumente/Dissertations/di ss_adusei-poku.pdf

Aitken, C. \& Taroni, F. (2004). Statistics and the Evaluation of Evidence for Forensic Scientists (Series Statistics in Practice) $\left(2^{a}\right.$ ed.).. West Sussex: Wiley.

Andrés-Pueyo, A. \& Echeburúa, E. (2010). Valoración del riesgo de violencia: Instrumentos disponibles e indicaciones de aplicación. Psicothema, 22, 403-409.

Andrews, D. \& Bonta, J. (1998). The psychology of criminal conduct ( $2^{\mathrm{a}}$ ed.), Cincinnati, $\mathrm{OH}$ : Anderson.

Andrews, D. \& Bonta, J. (2010). The psychology of criminal conduct ( $5^{\mathrm{a}}$ ed.), Cincinnati: Anderson Publishing Co.

Bayes, T. (1763). An Essay towards Solving a Problem in the Doctrine of Chances. Philosophical Transactions of the Royal Society, 53, 370-418.

Cruz-Ramírez, N., Acosta-Mesa, H. G., Carrillo-Calvet, H., Nava-Fernández, L. A. \& Barrientos-Martínez, R. E. (2007). Diagnosis of breast cancer using Bayesian Networks: A case study. Computers in Biology and Medicine, 37, 1553-1564.

Fenton, N. \& Neil, M. (2010). Comparing risks of alternative medical diagnosis using Bayesian arguments. Journal of Biomedical Informatics, 43, 45-495.

Gendreau, P., Little, T. \& Goggin, C. (1996). A meta-analysis of the predictors of adult offender recidivism: What works!. Criminology, 34(4), 575-607.

Grubin, D. \& Wingate, S. (1996). Sex offence recidivism: prediction versus understanding. Criminal Behavior and Mental Health, 6, 349-359.

Hanson, R. K. (1998). What do we know about sex offender risk assessment? Psychology, Public Policy, and Law, 4, 50-72.

Hanson, R. K. \& Bussière, M. T. (1998). Predicting relapse: a meta-analysis of sexual offender recidivism studies. Journal of Consulting and Clinical Psychology, 66 (2), 348-362.

Hanson, R. K. \& Morton-Bourgon, K. (2004). Predictors of sexual recidivism: an updated meta-analysis. Department of the Solicitor General Canada.

Hanson, R. K., \& Morton-Bourgon, K. E. (2005). The characteristics of persistent 
sexual offenders: A meta-analysis of recidivism studies. Journal of Consulting and Clinical Psychology, 73, 1154-1163.

Harris, A. J. R. \& Hanson, R. K. (2004). Sex offender redidivism: A simple Question. Department of the Solicitor General Canada.

La Fond, J. Q. (2005). Preventing sexual violence: How society should cope with sex offenders. Washington, D.C.: American Psychological Association.

Lalumière, M. L. \& Quinsey, V. L. (1996). Sexual deviance, antisociality, mating effort, and the use of sexually coercive behaviours. Personal individual differences, 21(1), 33-48.

Langevin, R., Curnoe, S., Fedoroff, P., Bennett, R., Langevin, M., Peever, C., Pettica, R. \& Sandhu, S. (2006). Lifetime Sex Offender Recidivism: A 25-Year FollowUp Study Canadian Journal of criminology and criminal justice, 46 (5), 531552.

Lee, C. \& Lee, K. J. (2006). Application of Bayesian Network to the probabilistic risk assessment of nuclear waste disposal, Reliability Engineering \& System Safety, $91(5), 515-532$.

Lösel, F. (2002). ¿Sirve el tratamiento para reducir la reincidencia de los delincuentes sexuales? En Redondo, S. (Coord.), Delincuencia sexual y sociedad. Barcelona: Ariel.

Murray, J. B. (2000). Psychological Profile of Pedophiles and Child Molesters. The Journal of Psychology, 134 (2), 211-224.

Olumide, K. M. (2010). A Probabilistic and Graphical Analysis of Evidence in O.J. Simpson's Murder Case. Tesis doctoral no publicada. Florida State University. Disponible en: http://diginole.lib.fsu.edu/etd/2287/

Pollino, C. A., Woodberry, O., Nicholson, A., Korb, K. \& Hart, B. T. (2007). Parameterisation and evaluation of a Bayesian network for use in an ecological risk assessment. Environmental Modelling and Software, 22, 1140-1152.

Quinsey, V. L.; Lalumière, M. L.; Rice, M. E. \& Harris, G. T. (1995). Predicting sexual offenses. En: Campbell, J. (Ed.). Assessing dangerousness: Violence by sexual offenders, batterers, and child abusers. Thousand Oaks, CA, EE.UU.: Sage Publications, Inc, 114-137.

Quinsey, V. L., Rice, M. E. \& Harris, G. T. (1995). Actuarial prediction of sexual recidivism. Journal of Interpersonal Violence, 10 (1), 85-105.

Redondo, S. (2006). ¿Sirve el tratamiento para rehabilitar a los delincuentes sexuales? Revista Española de Investigación Criminológica, 6, Art. 4.. Disponible en: http://www.criminologia.net/pdf/reic/ano4-2006/a42006art3.pdf 
Redondo, S. (2008). Individuos, sociedades y oportunidades en la explicación y prevención del delito: Modelo del Triple Riesgo Delictivo (TRD). Revista Española de Investigación Criminológica, 6, Art. 7. Disponible en: http://www.criminologia.net/pdf/reic/ano6-2008/a62008art7.pdf

Redondo, S. \& Garrido, V. (2008). Efficacy of a psychological treatment for sex offenders. Psicothema , 20(1), 4-9.

Roberts, C. F; Doren, D. M. \& Thornton, D. (2006). Dimensions Associated with Assessments of Sex Offender Recidivism Risk. Criminal Justice and Behavior, 29 (5), 569-589.

Soler, C. \& García, C. (2007). Análisis de las variables relacionadas con la reincidencia de los agresores sexuales. Documents de Treball, Investigació: Àmbit social $i$ criminològic. Centre d'estudis jurídics i formació especialitzada. Departament de Justícia. Generalitat de Catalunya.

Ticehurst, J. L., Newham, L. T. H., Rissik, D., Letcher, R. A. \& Jakeman, A. J. (2007). A Bayesian Network approach for assessing the sustainability of coastal lakes in New South Wales, Australia. Environmental Modelling and Software, 22 (8), 1129-1139.

Rosario Delgado es Profesora Titular de Estadística e Investigación Operativa en el Departamento de Matemáticas de la Universitat Autònoma de Barcelona (UAB) desde 1998. Licenciada en Matemáticas por la Universitat de Barcelona (UB) en el año 1987, obtuvo su doctorado en Matemáticas por la misma universidad en 1994. Su investigación en el campo de la Probabilidad y los Procesos Estocásticos empezó con el "Cálculo Estocástico", pero en la actualidad se dedica principalmente al estudio de la "Teoría de Colas" y a otras aplicaciones de la Probabilidad y la Estadística a diversas áreas del ámbito de las Ciencias y las Ciencias Sociales. Es miembro del Grupo de Análisis Estocástico de la UAB. Página web: http://gent.uab.cat/rosario_delgado/

Xavier-Andoni Tibau obtuvo su Grado en Ciencias Ambientales por la Universitat Autònoma de Barcelona (UAB) en el año 2010 y un Máster en Criminalística por la misma universidad en 2014. También es estudiante del Grado de Criminología de la Universitat Oberta de Catalunya (UOC). 


\section{Anexo 1}

Para ilustrar, en el caso más sencillo posible, el uso del Teorema de Bayes, vamos a considerar en este anexo sólo una variable explicativa: Versatilidad (V). A partir de la información del informe previo estimamos las probabilidades de esta variable condicionadas a los valores de la variable Reincidencia:

$$
\begin{gathered}
P(V=S i ́ / R=\text { Sexual })=0.643 \\
P(V=S i ́ / R=\text { Violenta })=0.529 \\
P(V=S i ́ / R=\text { General })=0.579 \\
P(V=S i ́ / R=\text { Sin reincidencia })=0.151
\end{gathered}
$$

Las probabilidades correspondientes de que $\mathrm{V}=\mathrm{No}$ son las complementarias y se obtienen restando de 1 las probabilidades de que V=Sí, ya que la variable V sólo puede tomar los valores "Sí" y "No". Recogemos estas probabilidades en la Tabla 6:

Tabla 6: Probabilidades condicionadas del nodo Versatilidad

\begin{tabular}{|l|c|c|c|c|}
\hline \multirow{2}{*}{ Versatilidad } & \multicolumn{4}{|c|}{ Reincidencia } \\
\cline { 2 - 5 } & Sexual & Violenta & General & Sin reincidencia \\
\hline Sí & $64.30 \%$ & $52.90 \%$ & $57.90 \%$ & $15.10 \%$ \\
\hline No & $35.70 \%$ & $47.10 \%$ & $42.10 \%$ & $84.90 \%$ \\
\hline
\end{tabular}

Centrándonos en el caso de la reincidencia sexual, sabemos que el riesgo (o probabilidad) a priori de este tipo de reincidencia es $\mathrm{P}(\mathrm{R}=\mathrm{Sexual})=0.058$ (Tabla 1). Pero si consideramos un delincuente sexual para el que conocemos la evidencia E de que Versatilidad $=$ Sí, podemos recalcular su riesgo de reincidencia sexual a partir de esta evidencia, obteniendo el riesgo (o probabilidad) de reincidencia sexual a posteriori $\mathrm{P}(\mathrm{R}=$ Sexual/E), siendo E la evidencia $V=S i ́$, mediante el Teorema de Bayes:

$$
P(R=\text { Sexual } / E)=\frac{P(E / R=\text { Sexual }) P(R=\text { Sexual })}{P(E)} .
$$


En la fórmula anterior conocemos los valores de las dos probabilidades del numerador, que son $\mathrm{P}(\mathrm{E} / \mathrm{R}=$ Sexual $)=0.643$ (Tabla 6) y $\mathrm{P}(\mathrm{R}=\mathrm{Sexual})=0.058$ (Tabla 1). Aunque la probabilidad del denominador no la conocemos de entrada, la podemos calcular usando la Fórmula de las Probabilidades Totales. De esta forma:

$$
\begin{aligned}
\mathrm{P}(\mathrm{V}=\mathrm{S} i ́) & =\mathrm{P}(\mathrm{E})=\mathrm{P}(\mathrm{E} / \mathrm{R}=\text { Sexual }) \mathrm{P}(\mathrm{R}=\text { Sexual })+\mathrm{P}(\mathrm{E} / \mathrm{R}=\text { Violenta }) \mathrm{P}(\mathrm{R}=\text { Violenta }) \\
& +\mathrm{P}(\mathrm{E} / \mathrm{R}=\text { General }) \mathrm{P}(\mathrm{R}=\text { General })+\mathrm{P}(\mathrm{E} / \mathrm{R}=\text { Sin reincidencia }) \mathrm{P}(\mathrm{R}=\text { Sin reincidencia }) \\
& =0.643 \times 0.058+0.529 \times 0.065+0.579 \times 0.062+0.151 \times 0.815=0.230642
\end{aligned}
$$

Resulta más sencillo realizar este cálculo usando software especializado. Para ello, introducimos la información de las Tablas 1 y 6 en el programa Hugin Lite, y obtenemos el cálculo de $\mathrm{P}(\mathrm{V}=\mathrm{S} i ́)$ (y su complementario, $\mathrm{P}(\mathrm{V}=\mathrm{No})$ ), en porcentajes, como podemos ver en la Figura 6.

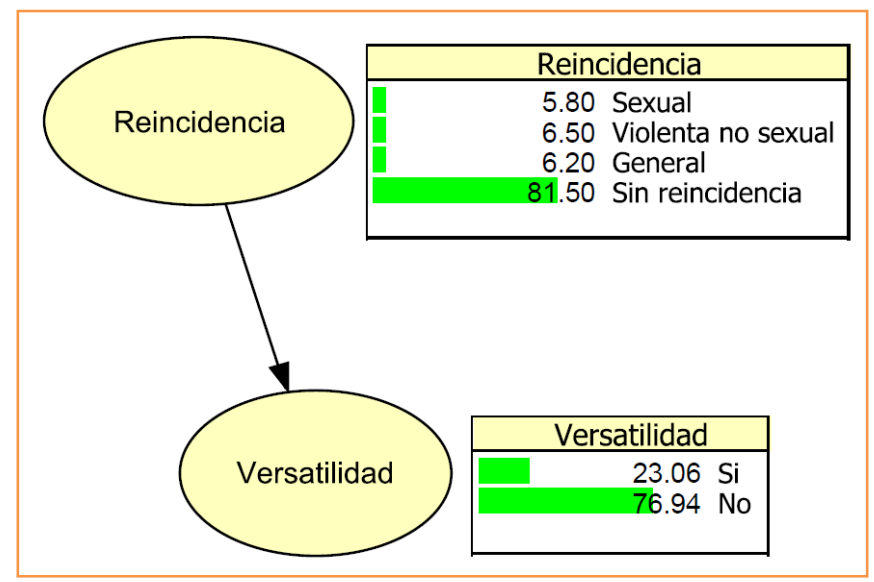

Figura 6. Grafo y probabilidades de la Red Bayesiana con dos nodos.

Sustituyendo el valor $\mathrm{P}(\mathrm{E})=0.230642$ en el denominador de la fórmula (1) tenemos que

$$
\begin{gathered}
P(R=\text { Sexual } / E)=\frac{P(E / R=\text { Sexual }) P(R=\text { Sexual })}{P(E)}=\frac{0.643 \times 0.058}{0.230642} \\
=\frac{0.037294}{0.230642}=0.161696482
\end{gathered}
$$

En forma esquemática:

$$
\begin{array}{r}
\text { Riesgo de Reincidencia Sexual a priori }=5.8 \%+\text { Evidencia }(V=S 1 ́) \\
=\text { Riesgo de Reincidencia Sexual a posteriori }=16.17 \%
\end{array}
$$


Este procedimiento, que permite obtener la probabilidad a posteriori a partir de la probabilidad a priori y de una evidencia, se conoce como Propagación Bayesiana.

El riesgo de reincidencia sexual a posteriori, que hemos calculado usando el Teorema de Bayes a partir de la evidencia, es decir, mediante la Propagación Bayesiana, también se puede obtener muy fácilmente a partir de la Red Bayesiana con el programa Hugin Lite como vemos en la Figura 7, donde además aparecen el resto de riesgos de reincidencia (violenta y general) y la tasa de no reincidencia.

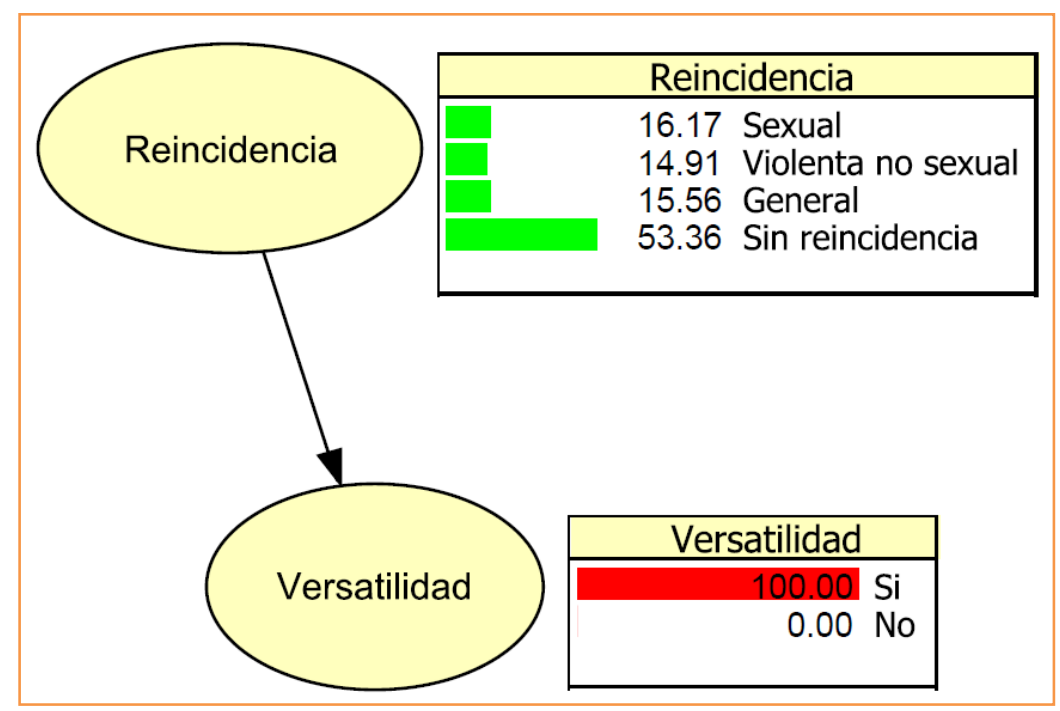

Figura 7. Riesgo de reincidencia a posteriori con Evidencia: Versatilidad=Sí. 